\title{
ANTIBACTERIAL CAPACITY AND IDENTIFICATION OF BIOACTIVE COMPOUNDS BY GCMS OF ALLIUM CEPA
}

\author{
DUSHYANT SHARMA, REENA RANI, MONIKA CHATURVEDI, J. P. YADAV* \\ Department of Genetics, Maharshi Dayanand University, Rohtak, Haryana, India 124001 \\ Email: yadav1964@rediffmail.com
}

Received: 16 Nov 2017 Revised and Accepted: 21 Dec 2017

\begin{abstract}
Objective: Plants offer a novel source for the isolation of a wide variety of medicinal agents. Allium cepa commonly known as onion is very well known medicinal plants and we investigated the antibacterial activity of different extracts and their phytochemical analysis by gas chromatography mass spectrometry (GCMS).

Methods: The extracts of $A$. cepa prepared in six different solvents was analyzed for antibacterial activity against nine American type cell culture (ATCC) reference bacterial strains i.e. Shigella flexneri, Enterococcus faecalis, Staphylococus aureus, Proteus mirabilis, Salmonella typhi, Serratia marcescens, Klebsiella pneumonia, Escherichia coli, Pseudomonas aeruginosa by using the agar well diffusion method. GCMS analysis also has been carried out for their phytochemical analysis.
\end{abstract}

Results: The results obtained from agar well diffusion assay showed the zone of inhibition range from $10 \pm 0.76$ to $26 \pm 0.76$ mm for different extracts. The methanol extract was found most potent against $K$. pneumonia and $S$. marcescens with the zone of inhibition of $26 \pm 0.76$ mm for both strains. Minimum inhibitory concentration (MIC) values were in the range of 1.87 to $7.5 \mathrm{mg} / \mathrm{ml}$ and the MIC values for K. pneumonia and S. marcescens was 1.87 $\mathrm{mg} / \mathrm{ml}$. A total of 43 compounds were identified by GCMS analysis. Out of them dodecanoic acid was found common in all extracts.

Conclusion: It is concluded that Allium cepa have good antibacterial activity so it can be used for the treatment of various infectious diseases.

Keywords: Antibacterial activity, GCMS, Allium cepa, Bioactive compounds

(C) 2018 The Authors. Published by Innovare Academic Sciences Pvt Ltd. This is an open access article under the CC BY license (http://creativecommons.org/licenses/by/4.0/) DOI: http://dx.doi.org/10.22159/ijpps.2018v10i2.23698

\section{INTRODUCTION}

Medicinal plants are being used for the treatment of different kind of diseases since ancient times. Plants synthesize a number of chemical compounds which are not directly involved in plant growth, but responsible for the different biological activities and provide protection from predators such as insects, fungi and herbivorous [1]. These chemicals are known as secondary metabolites. The secondary metabolites isolated from plants act in the human body in the similar way of the chemical compounds of allopathic drugs in their mechanism of action [2] Moreover, day by day the pathogens are being resistant against the synthetic drug, due to which herbal medicines can be an effective source for treatment of diseases with lesser side effects [3-4].

A. сера has been used as spices, vegetables, ornamentals and as medicines for curing and treatment of various diseases. The Allium genus comprises of more than 700 species, widely distributed all over the world [5] and known for their flavor, easy growth and long storage time. It is one of the civilization's oldest medicines and described as the dynamite of natural foods. Allium species are characterized by their rich content of sulfur compounds that are responsible for their organoleptic characteristics [6-7] and contributes to the antioxidant and antimicrobial activities [8]. Many studies have been done for its uses in the treatment of different diseases. The bulb is the main and most commonly used part of the onion. The bulb of $A$. сеpa has been reported to possess various activities like antihelmintic, antibacterial, anti-inflammatory, antiseptic, antispasmodic, carminative, diuretic, expectorant, febrifuge, hypoglycemic, hypotensive, lithintropic, stomachic and tonic [9-11]. Quercetin is one of the major flavanol beside other phytochemicals present in the $A$. серa which has antioxidant, antibacterial, urease inhibition and anti melanogenesis activities [12-13]. Diallyl disulphide, polyphenols and anthocyanins are the other major components present in A. cepa [14].

Keeping in view of the importance of this plant, the present study has been carried out to check the antibacterial activity of different extracts of $A$. cepa against different bacteria. GCMS analysis also has been carried out for their phytochemical analysis.

\section{MATERIALS AND METHODS}

Chemicals and reagents

Nutrient agar, peptone water and streptomycin discs were purchased from HiMedia, India. Resazurin was purchased from Sigma-Aldrich Chemicals Private Limited, India. The solvents used for the preparation of plant extracts were purchased from sisco research laboratory (SRL), India. All solvents and chemicals, purchased were of analytical grade.

\section{Preparation of plant extracts}

Onions (red type) were purchased from the local market of Rohtak $\left(28.8909^{\circ} \mathrm{N}\right.$ and $\left.76.5796^{\circ} \mathrm{E}\right)$, Haryana, India. These were peeled off, followed by washing and subjected to shade dry. The dried material was ground in an electrical grinder to obtain powder form. The powdered material $(50 \mathrm{gms})$ was extracted with six organic solvents i.e. acetone, benzene, chloroform, ethyl acetate, methanol, and petroleum ether $(1: 10)$ using cold percolation for 48-72 h. The obtained extracts were filtered using Whatman No. 1 filter paper and then concentrated using rotary evaporator at $40^{\circ} \mathrm{C}$.

\section{Antibacterial activity}

The antibacterial activity of the extracts was analyzed against nine different american type cell culture (ATCC) reference bacterial strains i.e. Shigella flexneri ATCC 12022, Enterococcus faecalis ATCC 29212, Staphylococus aureus ATCC 259323, Proteus mirabilis ATCC 43071, Salmonella typhi ATCC 13311, Serratia marcescens ATCC 27137, Klebsiella pneumonia ATCC 700603, Escherichia coli ATCC 25922 Pseudomonas aeruginosa ATCC 27853 by using agar well diffusion method of Perez et al. (1990) with slight modifications [15]. The bacterial strains were obtained from Department of microbiology of Pt. B. D. Sharma University of Health Sciences, Rohtak, Haryana, India.

\section{Minimum inhibitory concentration (MIC)}

MIC was determined using micro broth dilution method using 96 multi-well microtitre plates following the method of Sarker et al. 
(2007) with slight modifications [16]. Resazurin was used as indicator dye $\left(270 \mathrm{mg} / 40 \mathrm{ml} \mathrm{dH_{2 } \mathrm { O }}\right)$, purple colour of indicator dye reduced in the presence of living bacteria into pink/colourless. In the absence of living bacteria the colour of the indicator remains purple. The lowest concentration at which colour change occurred was taken as MIC.

\section{GCMS analysis}

Phytochemical analysis of all six extracts of A. cepa was carried out using GCMS analyzer (BRUKER SCION 436-GC SQ). Extracts were dissolved in methanol (HPLC grade) and filtered through Whatman ${ }^{\mathrm{TM}}$ FILTER DEVICE $(0.2 \mu \mathrm{m})$. Helium $(99.99 \%)$ was used as carrier gas, at flow rate of $1 \mathrm{ml}$ per minute in split mode. RESTEK Rtx ${ }^{\circledR}-5$ (Crossbond ${ }^{\circledR}$ 5\% diphenyl/95\% dimethyl polysiloxane) with $30 \mathrm{~m}$ length, $0.25 \mu \mathrm{m}$ df and $0.25 \mathrm{~mm}$ ID column was used for separation of phytochemicals. $2 \mu \mathrm{l}$ of the sample was injected to the column. The injector temperature was $280^{\circ} \mathrm{C}$. The temperature of oven was started at $70{ }^{\circ} \mathrm{C}$ and hold for $2 \mathrm{~min}$ and then raised at a rate of $7{ }^{\circ} \mathrm{C}$ per minute up to $320^{\circ} \mathrm{C}$; hold for $1 \mathrm{~min}$. Temperature of ion sources was maintained at $250^{\circ} \mathrm{C}$. The mass spectrum obtained by electron ionization at $70 \mathrm{eV}$ and detector operates in scan mode 30 to $500 \mathrm{Da}$ atomic units. Total running time was $38.71 \mathrm{~min}$ including 3 min solvent delay.

\section{RESULTS AND DISCUSSION}

\section{Yield of extracts}

The measured yield of the extracts and \% age of extracted values have been given in table 1 .

Table 1: The yield of $A$. cepa extracts and \%age of extracted value

\begin{tabular}{llll}
\hline S. No. & Extracts & Yield of extracts (g) & \%age of extracted value (Quantity of extract obtained $\times$ 100/Weight of dried powder) \\
\hline 1 & Methanol & 5.68 & 11.36 \\
2 & Acetone & 4.69 & 9.38 \\
3 & Ethyl acetate & 3.45 & 6.9 \\
4 & Chloroform & 0.870 & 1.5 \\
5 & Benzene & 0.746 & 1.4 \\
6 & Petroleum ether & 0.480 & 0.96 \\
\hline
\end{tabular}

\section{Antibacterial activity}

In the present study antimicrobial activities of extracts of $A$. cepa was investigated against reference bacterial strains at different concentrations by agar well diffusion assay. All the extracts showed good antibacterial activity with the zone of inhibition diameter ranging from 10 to $26 \mathrm{~mm}$ for different bacteria (table 2). The methanol extract showed highest antibacterial activity at all concentration as compared to other different extracts. Methanol extract of $A$. сера showed concentration dependent activity. The concentration of $40 \mathrm{mg} / \mathrm{ml}$ was found to be more effective. Highest zone of inhibition was reported against K. pneumonia ATCC 700603 and $S$. marcescens ATCC 27137. Similarly, acetone extract showed 17 $\mathrm{mm}$ and $15 \mathrm{~mm}$ zone of inhibition against S. flexneri ATCC 12022 and E. coli ATCC 25922 respectively. The outcomes of our study indicate that $A$. cepa have significant antimicrobial potential which supports the study of Palaksha et al., [17]. It has been reported that the antimicrobial activity of $A$. сеpa due to the presence of organosulfur and phenolic compounds [18].

Antibacterial activity of extracts of $A$. cepa has been analyzed against Bacillus subtillis and $S$. aureus by Sable et al. and the zone of inhibition measured were $8 \mathrm{~mm}$ and $9 \mathrm{~mm}$ respectively. A. cepa extract in combination with Zingiber officinale extract showed the zone of inhibition of $13 \mathrm{~mm}$ and $11 \mathrm{~mm}$ against B. subtillis and $S$. aureus respectively [19]. Thus, the synergistic effects may increase the potential of the A. cepa. Antagonistic effect of the onion extracts has been checked against bacterial isolates i.e. E. coli, S. aureus, Streptococcus pneumonia and Streptococcus pyogenes with the inhibition zone of $17,19,17$ and $20 \mathrm{~mm}$ respectively [20]. Kim et al. have studied the effect of $A$. серa extracts on the oral pathogenic bacteria i.e. Streptococcus mutans, Streptococcus sobrinus, Porphyromonas gingivalis and Prevotella intermedia and found that extracts were active against all of these bacteria [21]. Shakurfow et $a l$. have analyzed the antibacterial activity of cold water and organic solvent extracts against Listeria monocytogenes and found that the cold water extract was more effective than the organic solvent extract [22]. Santas et al. have checked the antibacterial activity of ethyl acetate extract of A. cepa against four Gram+ve (Bacillus cereus, S. aureus, Microcroccus luteus and Listeria monocytogenes) and two Gram-ve bacteria (E. coli and P. aeruginosa).

They observed that the extract inhibit the Gram+ve bacteria effectively while Gram-ve were found resistant [23]. On the contrary, in the present study the methanol extract of $A$. cepa was more effective against the Gram-ve bacteria.

Table 2: The zone of inhibition in $\mathrm{mm}$ against different bacterial strains

\begin{tabular}{|c|c|c|c|c|c|c|c|}
\hline Bacterial strain & Methanol & Acetone & Ethyl acetate & Chloroform & Petroleum ether & Benzene & Streptomycin \\
\hline S. flexneri & $20 \pm 0.76^{*}$ & $17 \pm 1.00$ & $10 \pm 0.57$ & - & $14 \pm 0.57$ & - & $21 \pm 0.57$ \\
\hline E. feacalis & $14 \pm 0.57$ & $13 \pm 1.00$ & $10 \pm 0.76$ & $11 \pm 1.00$ & $12 \pm 1.00$ & $11 \pm 0.57$ & $23 \pm 0.57$ \\
\hline S. aureus & $16 \pm 0.76$ & - & $12 \pm 0.76$ & - & $12 \pm 1.00$ & - & $26 \pm 0.76$ \\
\hline P. mirabilis & $20 \pm 0.57$ & - & - & $11 \pm 0.57$ & - & $11 \pm 0.76$ & $23 \pm 1.00$ \\
\hline S. typhi & $15 \pm 1.00$ & $12 \pm 0.57$ & - & - & - & - & $19 \pm 1.00$ \\
\hline S. marcescens & $26 \pm 0.76$ & $11 \pm 0.76$ & $10 \pm 1.00$ & $12 \pm 0.57$ & $10 \pm 0.76$ & $12 \pm 0.76$ & $20 \pm 0.57$ \\
\hline K. pneumonia & $26 \pm 0.76$ & $10 \pm 0.57$ & $17 \pm 1.00$ & - & $10 \pm 0.57$ & - & $19 \pm 0.57$ \\
\hline E. coli & $23 \pm 0.76$ & $10 \pm 0.57$ & $12 \pm 0.57$ & $10 \pm 0.76$ & - & - & $18 \pm 0.57$ \\
\hline$P$. aeruginosa & $20 \pm 0.57$ & $15 \pm 1.00$ & $10 \pm 0.57$ & $12 \pm 0.57$ & - & - & $23 \pm 0.76$ \\
\hline
\end{tabular}

*The zone of inhibition showed as mean \pm Standard deviation $(n=3)$

MIC values of different extracts against tested microbes have been shown in table 3 . The results showed that MIC values of $A$. серa extracts varied from $1.87 \mathrm{mg} / \mathrm{ml}$ to $7.50 \mathrm{mg} / \mathrm{ml}$. Lowest MIC value was observed for methanol extract $(1.87 \mathrm{mg} / \mathrm{ml})$ against $K$. pneumoniae and $S$. marcescens. It means that $A$. cepa methanol extract possess the highest antimicrobial activity as compared to other extracts. 
Table 3: MIC values (in $\mathrm{mg}$ ) of different extracts against different bacteria

\begin{tabular}{|c|c|c|c|c|c|c|}
\hline Bacterial strain & Methanol & Acetone & Ethyl acetate & Chloroform & Petroleum ether & Benzene \\
\hline S. flexneri & 5.0 & 3.75 & 7.5 & - & 3.75 & - \\
\hline E. feacalis & 3.75 & 5.0 & 7.5 & 7.5 & 5.0 & 5.0 \\
\hline S. aureus & 7.5 & - & 5.0 & - & 5.0 & - \\
\hline P. mirabilis & 3.75 & - & - & 7.5 & - & 5.0 \\
\hline S. typhi & - & 7.5 & - & - & - & - \\
\hline S. marcescens & 1.87 & 7.5 & 7.5 & 5.0 & 7.5 & 5.0 \\
\hline K. pneumonia & 1.87 & 7.5 & 3.5 & - & 7.5 & - \\
\hline E. coli & 7.5 & 7.5 & 5.0 & 7.5 & - & - \\
\hline P. aeruginosa & 5.0 & 5.0 & 3.75 & 5.0 & - & - \\
\hline
\end{tabular}

The MIC values are showed as mean $(n=3)$

\section{GCMS analysis}

Organic compounds of different extracts were identified by GCMS analysis and spectra result was matched with the National Institute of Standards and Technology (NIST) MS library. The lists of identified phytochemicals from different extracts (methanol, acetone, ethyl acetate, chloroform, benzene, petroleum ether) have been given in table 4 . The main phytochemicals identified by matching the spectra with NIST library were dodecanoic acid, methyl tetradecanoate, tetradecanoic acid, pentadecanoic acid, 14methyl-, methyl ester, methyl stearate, eicosanoic acid, methyl ester, di(2-ethylhexyl)adipate, 9-octadecenamide, (Z), 9-octadecenamide, (Z), tetracontane, 3,5,24-trimethyl, beta.-sitosterol. The spectra of the GCMS are given in fig. 1-6. The GCMS analysis revealed the presence of fatty acids and esters. Many fatty acids isolated from plants have been reported for their antimicrobial activity. Dodecanoic acid, also known as lauric acid has been isolated from coconut oil possesses good antibacterial activity against $S$. aureus, Bacillus cereus, Salmonella thypimurium and E. coli [24]. Pthalic acid, also known as benzoic acid has been tested against bacterial strain and zone of inhibition were ranged from 15 to $18 \mathrm{~mm}$ [25]. Odiba et $a l$. had extracted the beta sitostirol from honey bee Propolis and studied its antibacterial activity against P. aeruginosa, E. coli, $K$. pneumonia, S. aureus, Streptococcus pyrogenes, Corynebacterium ulcerans, Bacillus subtillis, Shigella dysentariae, P. mirabilis, Candida albicans, Candida krusei and Candida tropicalis. They concluded that beta sitosterol showed the good antibacterial activity [26]. We have also reported the presence of beta sitosterol in A. cepa extracts. The GCMS analysis of extracts showed the existence of volatile compounds which are comparable to the study done by Lekshmi $e$ t al. [27]. The similar compounds reported in our study are octadecanoic acid, undecane, sitosterol, tetradecanal, dibutyl phthalate etc. Farag et al. identified the 39 volatile compounds from A. cepa and A. sativum using solid-phase micro-extraction coupled to GCMS and 38 non-volatile compounds by using UPLC/PDA/orbitrapMS in methanol extracts [28]. In our study, most of the compounds identified in all six extracts were almost similar. However their quantities may differ as revealed from peak area and \% of total. Maximum numbers of compounds (25) were identified in chloroform extract using NIST library.

Table 4: Phytoconstituents of six extracts screened by GCMS analysis with area and \% of total

\begin{tabular}{|c|c|c|c|c|c|c|c|c|c|c|c|c|c|}
\hline \multirow[t]{2}{*}{ Name of compounds } & \multicolumn{6}{|l|}{ Area } & \multicolumn{6}{|c|}{$\%$ of total } & \multirow[t]{2}{*}{ Formula } \\
\hline & ACM & ACA & ACEA & ACC & ACPE & ACB & ACM & ACA & ACEA & ACC & ACPE & ACB & \\
\hline $\begin{array}{l}\text { Dodecanoic acid, } \\
\text { methyl ester }\end{array}$ & $6.501 \mathrm{e}+7$ & $6.490 \mathrm{e}+7$ & $5.116 \mathrm{e}+7$ & $6.910 \mathrm{e}+7$ & $5.934 \mathrm{e}+7$ & $7.648 \mathrm{e}+7$ & 10.016 & 12.909 & 7.422 & 6.798 & 8.650 & 6.284 & $\mathrm{C}_{13} \mathrm{H}_{26} \mathrm{O}_{2}$ \\
\hline Dodecanoic acid & $2.912 \mathrm{e}+8$ & $4.592 \mathrm{e}+7$ & $9.358 \mathrm{e}+7$ & $1.230 \mathrm{e}+8$ & $7.029 \mathrm{e}+7$ & $1.293 e+8$ & 44.860 & 9.133 & 13.576 & 12.097 & 10.247 & 10.626 & $\mathrm{C}_{12} \mathrm{H}_{24} \mathrm{O}_{2}$ \\
\hline $\begin{array}{l}\text { Methyl } \\
\text { tetradecanoate }\end{array}$ & $3.041 \mathrm{e}+7$ & $3.224 \mathrm{e}+7$ & - & $3.763 \mathrm{e}+7$ & - & - & 4.685 & 6.414 & - & 3.702 & - & - & $\mathrm{C}_{15} \mathrm{H}_{30} \mathrm{O}_{2}$ \\
\hline Tetradecanoic acid & $5.040 \mathrm{e}+7$ & $1.441 \mathrm{e}+7$ & - & $1.505 \mathrm{e}+7$ & $1.088 \mathrm{e}+7$ & - & 7.766 & 2.867 & - & 1.481 & 1.587 & - & $\mathrm{C}_{14} \mathrm{H}_{28} \mathrm{O}_{2}$ \\
\hline $\begin{array}{l}\text { Phthalic acid, hex-3-yl } \\
\text { isobutyl ester }\end{array}$ & $7.923 e+6$ & - & - & - & - & - & 1.221 & - & - & - & - & - & $\mathrm{C}_{18} \mathrm{H}_{26} \mathrm{O}_{4}$ \\
\hline $\begin{array}{l}\text { Pentadecanoic acid, } \\
\text { 14-methyl-, methyl } \\
\text { ester }\end{array}$ & $6.338 \mathrm{e}+7$ & $7.851 \mathrm{e}+7$ & - & - & $4.510 \mathrm{e}+6$ & - & 9.766 & 15.617 & - & - & 0.657 & - & $\mathrm{C}_{17} \mathrm{H}_{34} \mathrm{O}_{2}$ \\
\hline $\begin{array}{l}\text { 9,12-Octadecadienoic } \\
\text { acid, methyl ester, } \\
(\mathrm{E}, \mathrm{E})\end{array}$ & $5.744 \mathrm{e}+7$ & - & $1.964 \mathrm{e}+8$ & - & - & - & 8.850 & - & 28.492 & - & - & - & $\mathrm{C}_{19} \mathrm{H}_{34} \mathrm{O}_{2}$ \\
\hline Methyl stearate & $1.053 \mathrm{e}+7$ & - & $1.966 \mathrm{e}+7$ & $2.231 \mathrm{e}+7$ & $20.22 \mathrm{e}+7$ & $2.555 \mathrm{e}+7$ & 1.622 & - & 2.853 & 2.195 & 2.948 & 2.099 & $\mathrm{C}_{19} \mathrm{H}_{38} 0_{2}$ \\
\hline $\begin{array}{l}\text { Tetracontane, 3,5,24- } \\
\text { trimethyl }\end{array}$ & $8.674 \mathrm{e}+6$ & - & $9.829 e+6$ & - & - & - & 1.336 & - & 1.426 & - & - & - & $\mathrm{C}_{43} \mathrm{H}_{88}$ \\
\hline 1-Decanol, 2-hexyl & $1.006 \mathrm{e}+7$ & - & - & - & - & $3.214 \mathrm{e}+7$ & 1.550 & - & - & - & - & 2.641 & $\mathrm{C}_{16} \mathrm{H}_{34} \mathrm{O}$ \\
\hline $\begin{array}{l}\text { Eicosanoic acid, } \\
\text { methyl ester }\end{array}$ & $5.628 \mathrm{e}+6$ & - & $1.531 \mathrm{e}+7$ & $1.244 \mathrm{e}+7$ & - & $2.582 \mathrm{e}+7$ & 0.867 & - & 2.221 & 1.224 & - & 2.122 & $\mathrm{C}_{21} \mathrm{H}_{42} \mathrm{O}_{2}$ \\
\hline $\begin{array}{l}\mathrm{Di}(2- \\
\text { ethylhexyl)adipate }\end{array}$ & $7.241 \mathrm{e}+6$ & $5.209 \mathrm{e}+6$ & $7.188 \mathrm{e}+6$ & $7.489 e+6$ & $8.299 \mathrm{e}+6$ & - & 1.116 & 1.036 & 1.043 & 0.737 & 1.210 & - & $\mathrm{C}_{22} \mathrm{H}_{42} \mathrm{O}_{4}$ \\
\hline $\begin{array}{l}\text { Bis(2-ethylhexyl) } \\
\text { phthalate }\end{array}$ & $2.558 \mathrm{e}+7$ & $2.605 e+7$ & $2.952 \mathrm{e}+7$ & $3.084 \mathrm{e}+7$ & $2.778 \mathrm{e}+7$ & $2.042 \mathrm{e}+7$ & 3.941 & 5.182 & 4.283 & 3.034 & 4.050 & 1.678 & $\mathrm{C}_{24} \mathrm{H}_{38} \mathrm{O}_{4}$ \\
\hline $\begin{array}{l}\text { 9-Octadecenamide, } \\
\text { (Z) }\end{array}$ & $1.561 \mathrm{e}+7$ & $2.899 \mathrm{e}+7$ & $1.143 \mathrm{e}+7$ & $1.402 \mathrm{e}+7$ & $9.950 \mathrm{e}+6$ & - & 2.405 & 5.766 & 1.658 & 1.379 & 1.451 & - & $\mathrm{C}_{18} \mathrm{H}_{35} \mathrm{NO}$ \\
\hline Phenol, 4-propyl & - & $1.179 \mathrm{e}+7$ & $2.214 \mathrm{e}+7$ & - & - & - & - & 2.346 & 3.212 & - & - & - & $\mathrm{C}_{9} \mathrm{H}_{12} \mathrm{O}$ \\
\hline $\begin{array}{l}\text { 13-Octadecenoic acid, } \\
\text { methyl ester }\end{array}$ & - & $1.179 \mathrm{e}+8$ & - & - & - & - & - & 23.454 & - & - & - & - & $\mathrm{C}_{19} \mathrm{H}_{36} \mathrm{O}_{2}$ \\
\hline $\begin{array}{l}\text { Hexadecanoic acid, } \\
15 \text {-methyl-, methyl } \\
\text { ester }\end{array}$ & - & $1.417 \mathrm{e}+7$ & - & $2.206 \mathrm{e}+7$ & - & - & - & 2.818 & - & 2.170 & - & - & $\mathrm{C}_{18} \mathrm{H}_{36} \mathrm{O}_{2}$ \\
\hline Hentriacontane & - & $9.817 \mathrm{e}+6$ & - & $1.016 \mathrm{e}+7$ & $1.505 \mathrm{e}+7$ & - & - & 1.953 & - & 0.999 & 2.194 & - & $\mathrm{C}_{31} \mathrm{H}_{64}$ \\
\hline Tritetracontane & - & $1.080 \mathrm{e}+7$ & $2.040 \mathrm{e}+7$ & $2.354 \mathrm{e}+7$ & - & - & - & 2.148 & 2.959 & 2.316 & - & - & $\mathrm{C}_{43} \mathrm{H}_{88}$ \\
\hline $\begin{array}{l}\text { Heneicosanoic acid, } \\
\text { methyl ester }\end{array}$ & - & $8.505 e+6$ & - & - & - & - & - & 1.692 & - & - & - & - & $\mathrm{C}_{22} \mathrm{H}_{44} \mathrm{O}_{2}$ \\
\hline Oleanitrile & - & $3.350 \mathrm{e}+7$ & - & - & - & - & - & 6.664 & - & - & - & - & $\mathrm{C}_{18} \mathrm{H}_{33} \mathrm{~N}$ \\
\hline Phenol, 2-propyl & - & - & $2.214 \mathrm{e}+7$ & - & - & - & - & - & 3.212 & - & - & - & $\mathrm{C}_{9} \mathrm{H}_{12} \mathrm{O}$ \\
\hline
\end{tabular}




\begin{tabular}{|c|c|c|c|c|c|c|c|c|c|c|c|c|c|}
\hline Tridecanoic acid, 12- & - & - & $3.185 \mathrm{e}+7$ & - & - & $4.079 \mathrm{e}+7$ & - & - & 4.621 & - & - & 3.351 & $\mathrm{C}_{15} \mathrm{H}_{30} \mathrm{O}_{2}$ \\
\hline $\begin{array}{l}\text { 1,2- } \\
\text { Benzenedicarboxylic } \\
\text { acid, bis(2- } \\
\text { methylpropyl) ester }\end{array}$ & - & - & $8.522 \mathrm{e}+6$ & - & $8.880 \mathrm{e}+6$ & - & - & - & 1.236 & - & 1.294 & - & $\mathrm{C}_{16} \mathrm{H}_{22} \mathrm{O}_{4}$ \\
\hline $\begin{array}{l}\text { Hexadecanoic acid. } \\
\text { methyl ester }\end{array}$ & - & - & $1.020 \mathrm{e}+8$ & - & - & $1.993 e+8$ & - & - & 14.804 & - & - & 16.374 & $\mathrm{C}_{17} \mathrm{H}_{34} \mathrm{O}_{2}$ \\
\hline $\begin{array}{l}\text { Hexadecanoic acid, } \\
\text { ethyl ester }\end{array}$ & - & - & - & - & $7.521 \mathrm{e}+6$ & - & - & - & - & - & 1.096 & - & $\mathrm{C}_{18} \mathrm{H}_{36} \mathrm{O}_{2}$ \\
\hline $\begin{array}{l}\text { (R)-(-)-14-Methyl-8- } \\
\text { hexadecyn-1-ol }\end{array}$ & - & - & $1.086 \mathrm{e}+7$ & - & - & - & - & - & 1.576 & - & - & - & $\mathrm{C}_{17} \mathrm{H}_{32} \mathrm{O}$ \\
\hline $\begin{array}{l}\text { Docosanoic acid, } \\
\text { methyl ester }\end{array}$ & - & - & $6.518 \mathrm{e}+6$ & - & - & - & - & - & 0.946 & - & - & - & $\mathrm{C}_{23} \mathrm{H}_{46} \mathrm{O}_{2}$ \\
\hline $\begin{array}{l}\text { 9,19-Cyclolanostan-3- } \\
\text { ol,acetate, (3. beta.) }\end{array}$ & - & - & $1.021 \mathrm{e}+7$ & $1.116 \mathrm{e}+7$ & $7.021 \mathrm{e}+6$ & $2.122 \mathrm{e}+7$ & - & - & 1.482 & 1.098 & 1.023 & 1.990 & $\mathrm{C}_{32} \mathrm{H}_{54} \mathrm{O}_{2}$ \\
\hline beta.-Sitosterol & - & - & $7.344 \mathrm{e}+6$ & $1.264 \mathrm{e}+7$ & $5.612 \mathrm{e}+6$ & $1.742 \mathrm{e}+7$ & - & - & 1.064 & 1.243 & 0.818 & 4.431 & $\mathrm{C}_{29} \mathrm{H}_{50} \mathrm{O}$ \\
\hline $\begin{array}{l}\text { 9,19-Cyclolanost-24- } \\
\text { en-3-ol, acetate }\end{array}$ & - & - & $1.562 \mathrm{e}+7$ & $2.231 \mathrm{e}+7$ & $1.651 \mathrm{e}+7$ & $4.632 \mathrm{e}+7$ & - & - & 2.267 & 2.195 & 2.407 & 3.806 & $\mathrm{C}_{32} \mathrm{H}_{52} \mathrm{O}_{2}$ \\
\hline $\begin{array}{l}\text { 2-Methoxy-4- } \\
\text { vinylphenol }\end{array}$ & - & - & - & $2.066 \mathrm{e}+7$ & - & - & - & - & - & 2.032 & - & - & $\mathrm{C}_{9} \mathrm{H}_{10} \mathrm{O}_{2}$ \\
\hline $\begin{array}{l}3(2 \mathrm{H}) \text {-Furanone, } 2- \\
\text { hexyl-5-methyl }\end{array}$ & - & - & - & $2.043 \mathrm{e}+7$ & - & - & - & - & - & 2.010 & - & - & $\mathrm{C}_{11} \mathrm{H}_{18} \mathrm{O}_{2}$ \\
\hline $\begin{array}{l}11,14- \\
\text { Octadecadienoic acid, } \\
\text { methyl ester }\end{array}$ & - & - & - & $3.144 \mathrm{e}+8$ & - & - & - & - & - & 30.929 & - & - & $\mathrm{C}_{19} \mathrm{H}_{34} \mathrm{O}_{2}$ \\
\hline $\begin{array}{l}\text { (Z)6,(Z)9- } \\
\text { Pentadecadien-1-ol }\end{array}$ & - & - & - & $1.565 \mathrm{e}+7$ & - & $1.600 \mathrm{e}+7$ & - & - & - & 1.539 & - & 2.958 & $\mathrm{C}_{15} \mathrm{H}_{28} \mathrm{O}$ \\
\hline $\begin{array}{l}\text { Pentadecanoic acid, } \\
\text { methyl ester }\end{array}$ & - & - & - & - & $5.645 \mathrm{e}+6$ & - & - & - & - & - & 0.823 & - & $\mathrm{C}_{16} \mathrm{H}_{32} \mathrm{O}_{2}$ \\
\hline $\begin{array}{l}\text { Tridecanoic acid, } 12- \\
\text { methyl-, methyl ester }\end{array}$ & - & - & $3.185 \mathrm{e}+7$ & - & $3.240 \mathrm{e}+7$ & - & - & - & 4.621 & - & 4.723 & - & $\mathrm{C}_{15} \mathrm{H}_{30} \mathrm{O}_{2}$ \\
\hline $\begin{array}{l}\text { Methyl 11- } \\
\text { hexadecenoate }\end{array}$ & - & - & - & - & $6.816 \mathrm{e}+6$ & - & - & - & - & - & 0.994 & - & $\mathrm{C}_{17} \mathrm{H}_{32} \mathrm{O}_{2}$ \\
\hline $\begin{array}{l}\text { Methyl 12,13- } \\
\text { tetradecadienoate }\end{array}$ & - & - & - & - & $2.272 \mathrm{e}+8$ & - & - & - & - & - & 33.121 & - & $\mathrm{C}_{15} \mathrm{H}_{26} \mathrm{O}_{2}$ \\
\hline Oleic Acid & - & - & - & - & $7.245 \mathrm{e}+6$ & - & - & - & - & - & 1.056 & - & $\mathrm{C}_{18} \mathrm{H}_{34} \mathrm{O}_{2}$ \\
\hline $\begin{array}{l}\text { Docosanoic acid, } \\
\text { ethyl ester }\end{array}$ & - & - & - & - & $1.494 \mathrm{e}+7$ & - & - & - & - & - & 2.178 & - & $\mathrm{C}_{24} \mathrm{H}_{48} \mathrm{O}_{2}$ \\
\hline $\begin{array}{l}912- \\
\text { Hexadecadienoic acid, } \\
\text { methyl ester }\end{array}$ & - & - & - & - & - & $5.011 \mathrm{e}+8$ & - & - & - & - & - & 41.171 & $\mathrm{C}_{17} \mathrm{H}_{30} \mathrm{O}_{2}$ \\
\hline $\begin{array}{l}\text { Hexadecanoic acid, 2- } \\
\text { hydroxy-1- } \\
\text { (hydroxymethyl)ethyl } \\
\text { ester }\end{array}$ & - & - & - & - & - & $4.221 \mathrm{e}+7$ & - & - & - & - & - & 3.469 & $\mathrm{C}_{19} \mathrm{H}_{38} \mathrm{O}_{4}$ \\
\hline
\end{tabular}

ACM-Allium cepa methanol extract, ACA-Allium cepa acetone extract, ACEA-Allium cepa ethyl acetate extract, ACC-Allium cepa chloroform extract, ACPE-Allium cepa petroleum ether extract, ACB-Allium cepa benzene extract

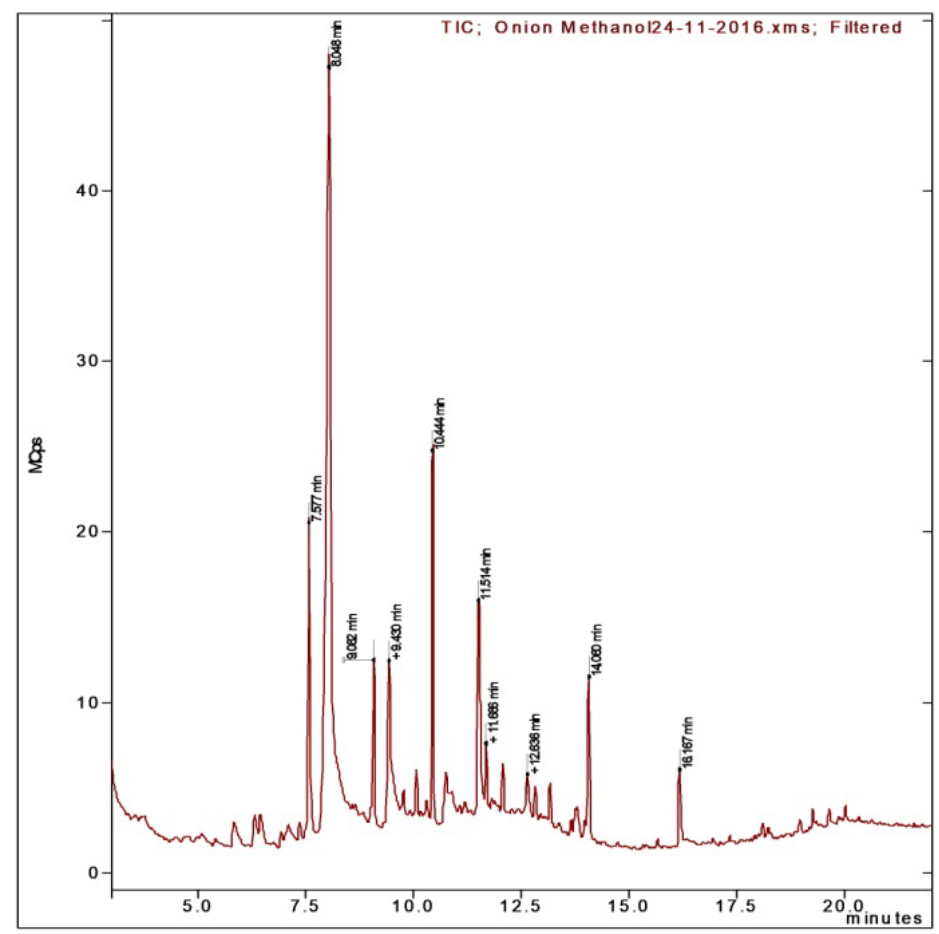

Fig. 1: GCMS spectra of methanol extract 


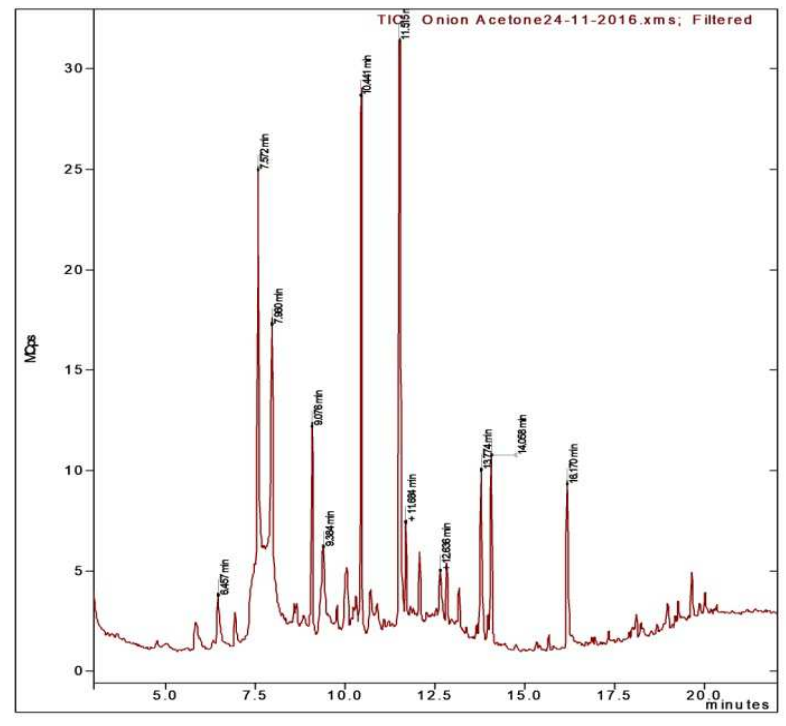

Fig. 2: GCMS spectra of Acetone extract

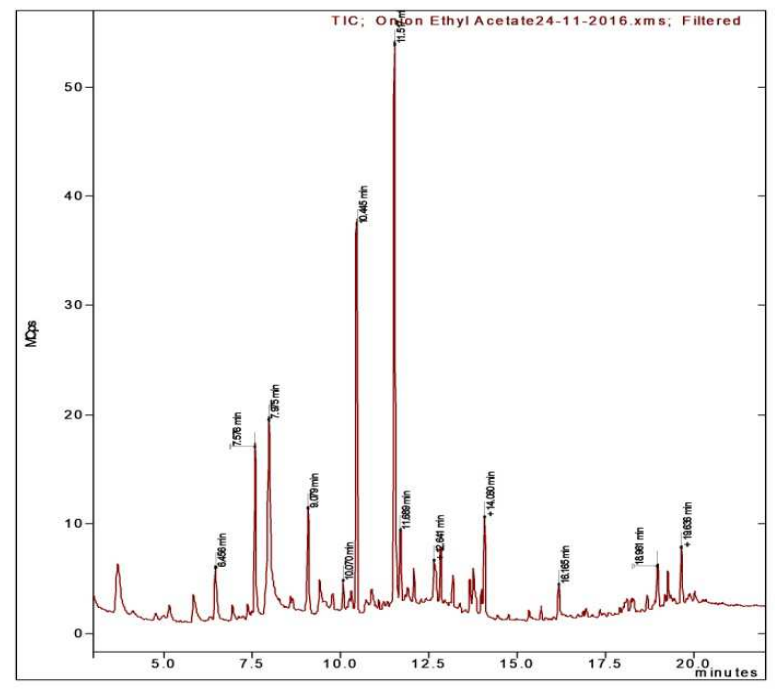

Fig. 3: GCMS spectra of ethyl acetate extract

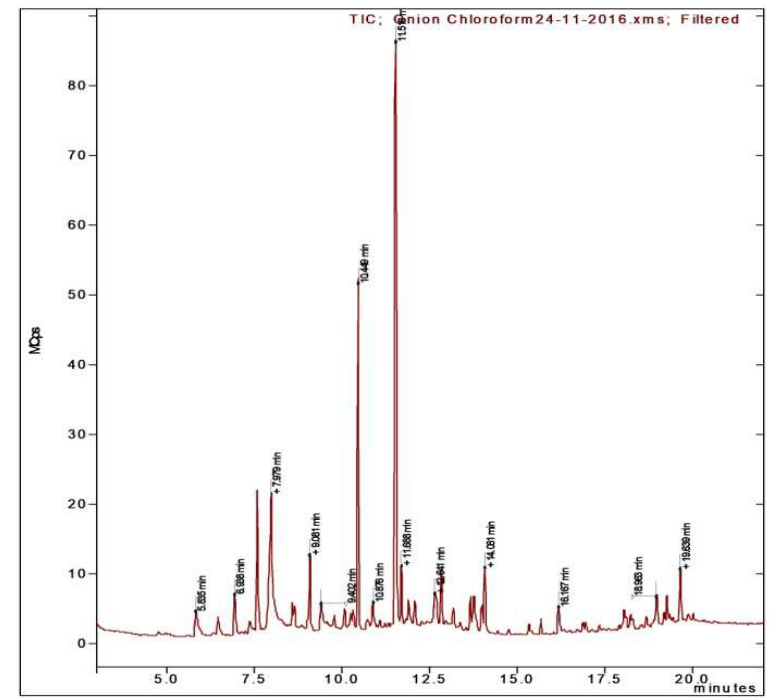

Fig. 4: GCMS spectra of chloroform extract

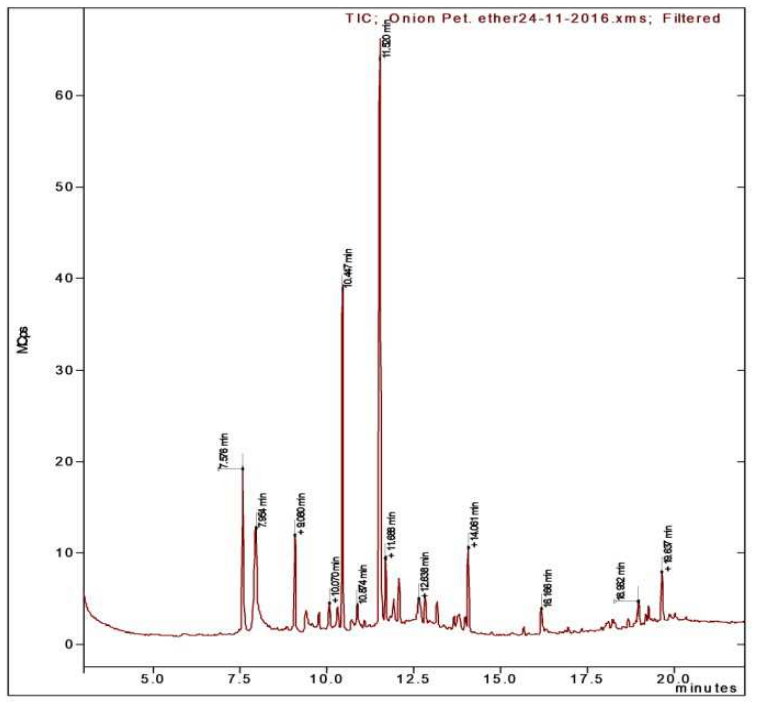

Fig. 5: GCMS spectra of petroleum ether extract

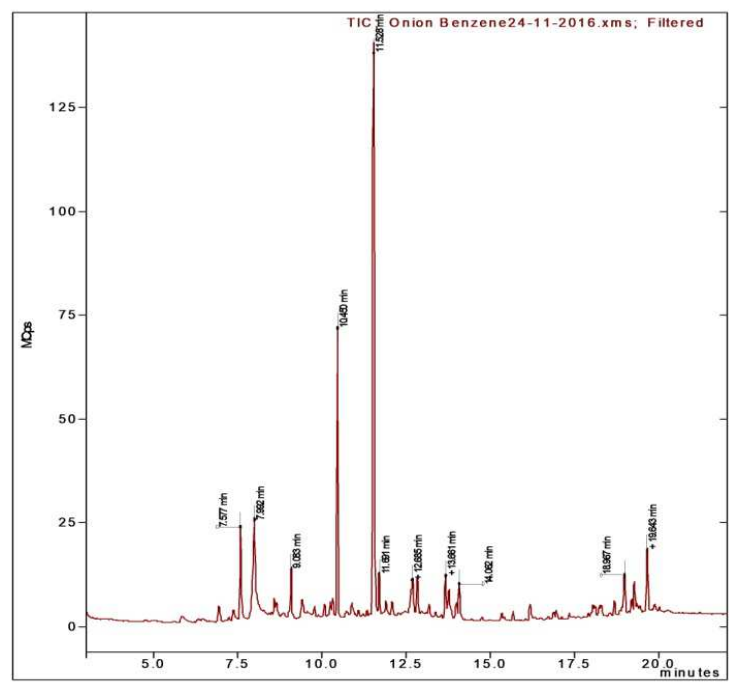

Fig. 6: GCMS spectra of Benzene extract

In this study, the common compounds identified were dodecanoic acid (methyl ester), dodecanoic acid and bis (2-ethylhexyl) phthalate in all the extracts. The maximum amount of dodecanoic acid was $44.86 \%$ in methanol extract. The \% of total dodecanoic acid (methyl ester) and bis (2-ethylhexyl) phthalate was comparable in all extracts which lies in the range of $6.284 \%$ to $12.91 \%$. The highest antibacterial activity was found in methanol extract of $A$. сеpa which may be due to the presence of higher amount of dodecanoic acid in comparison to other extracts. The highest antibacterial activity of methanol extract may also be due to the presence of volatile and non-volatile secondary metabolites. Further, research is required to study the effectiveness of purified active components from methanol extract of $A$. cepa.

\section{CONCLUSION}

It is concluded that A. cepa methanol extract has shown good antibacterial activity against different bacterial strains. GCMS analysis of different extracts revealed the presence of 43 phytochemicals. The findings of this work support the vision that this plant could provide biologically active natural drugs which may be useful for the treatment of bacterial infectious diseases.

\section{ACKNOWLEDGEMENT}

This research work was supported by grant from UGC BSR [F.41/2006 (BSR/7-371/2012] and UGC-SAP [F.20/2012(SAP-II)]. 


\section{ABBREVIATIONS}

ATCC: American Type Cell Culture, GCMS: Gas Chromatography Mass Spectrometry, MIC: Minimum Inhibitory Concentration.

\section{AUTHORS CONTIBUTIONS}

Dushyant Sharma has prformed experimentation work, data collection, and drafted the manuscript. Reena Rani has made significant involvement in the interpretation of data and revising the manuscript. Monika Chaturvedi participated in the design of the study and performed the statistical analysis. Jaya Parkash Yadav helped in designed the study and manuscript.

\section{CONFLICT OF INTERESTS}

There is no conflict of interest between authors.

\section{REFERENCES}

1. Pradeepa M, Kalidas V, Geetha N. Qualitative and quantitative phytochemical analysis and bactericidal activity of pelargonium graveolens L'her. Int J Appl Pharm 2016;8:7-11.

2. Ogunlowo OP, Arimah B, Adebayo M. Phytochemical analysis and comparison of in vitro antimicrobial activities of the leaf, stem bark and root bark of Ficus benghalensis. IOSR J Pharm 2013;3:33-8.

3. Sharma D, Yadav JP. An overview of phytotherapeutic approaches for the treatment of tuberculosis. Mini Rev Med Chem 2017;17:167-83.

4. Tyagi R, Sharma G, Jasuja ND, Menghani E. Indian medicinal plants as an effective antimicrobial agent. J Crit Rev 2017;3:69-71.

5. Keusgen M, Fritsch RM, Hisoriev H, Kurbonova PA, Khassanov FO. Wild Allium species (Alliaceae) used in folk medicine of Tajikistan and Uzbekistan. J Ethnobiol Ethnomed 2006;2:1-9.

6. Benkeblia N, Lanzotti V. Allium thiosulfinates: chemistry, biological properties and their potential utilization in food preservation. Food 2007;1:193-201.

7. Lanzotti V. The analysis of onion and garlic. J Chromatogr A 2006;1112:3-22.

8. Mnayer D, Fabiano-Tixier AS, Petitcolas E, Hamieh T, Nehme N, Ferrant C, et al. Chemical composition, antibacterial and antioxidant activities of six essentials oils from the Alliaceae family. Molecules 2014;19:20034-53.

9. Chinnala KM, Achant P, Vangala VL, Elsani MM. Evaluation for nephroprotective activity of ethanolic extract of Allium сера linn. In gentamicin-induced nephrotoxicity in rats. Asian J Pharm Clin Res 2017;10:356-9.

10. Jalaiah M, Sarvanan R, Gowtham $\mathrm{CH}$, Vinay $\mathrm{Y}$, Akhila $\mathrm{Y}$. Evaluation of cardioprotective activity of Allium cepa aerial leaves. Int J Curr Pharm Res 2017;9:27-30.

11. Almawlah YH, Alaa H, Al-Jelawi SO. Antibacterial activity of three plant extracts against multidrug resistance Pseudomonas aeruginosa. Asian J Pharm Clin Res 2017;10:193-7.

12. Shabana S, Kawai A, Kai K, Akiyama K, Hayashi H. Inhibitory activity against urease of quercetin glycosides isolated from Allium cepa and Psidium guajava. Biosci Biotechnol Biochem 2010;74:878-80.
13. Arung ET, Furuta S, Ishikawa H, Kusuma IW, Shimizu K, Kondo R. Anti-melanogenesis properties of quercetin-and its derivative-rich extract from Allium cepa. Food Chem 2011;124:1024-8.

14. Perez-Gregorio RM, Garcia-Falcon MS, Simal-Gandara J, Rodrigues AS, Almeida DP. Identification and quantification of flavonoids in traditional cultivars of red and white onions at harvest. J Food Comp Anal 2010;23:592-8.

15. Perez C, Pauli M, Bazerque P. An antibiotic assay by agar well diffusion method. Act Biol Med Exp 1990;15:113-5.

16. Sarker SD, Nahar L, Kumarasamy Y. Microtitre plate-based antibacterial assay incorporating resazurin as an indicator of cell growth, and its application in the in vitro antibacterial screening of phytochemicals. Methods 2007;42:321-4.

17. Palaksha MN, Banji D, Rao AS. In vitro evaluation of antibacterial activity of alcoholic extracts of ten South Indian spices against multi-resistant gram-positive and gram-negative bacteria by agar well diffusion method. World J Pharm Pharm Sci 2013;2:3840-7.

18. Griffiths G, Trueman L, Crowther T, Thomas B, Smith B. Onionsa global benefit to health. Phytother Res 2002;16:603-15.

19. Sable MG, Puttewar TY, Patil RY. Investigation of antibacterial activity of Allium cepa (onion) Zingiber officinale (Ginger). Int J Curr Res 2014;6:8768-78.

20. Shinkafi SA, Dauda H. Antibacterial activity of Allium Cepa (Onion) on some pathogenic bacteria associated with ocular infections. Schol J Appl Med Sci 2013;1:147-51.

21. Kim WJ, Lee KA, Kim KT, Chung MS, Cho SW, Paik HD. Antimicrobial effects of onion (Allium cepa L.) peel extracted by subcritical water. Food Sci Biotech 2011;20:1101-6.

22. Shakurfow FA, Buazzi MM, Gamal MA. Assessment of antimicrobial activity of onion (Allium cepa) and garlic (Allium sativum) extracts on Listeria monocytogenes; in vitro study. Lebda Med J 2016;1:1-5.

23. Santas J, Almajano MP, Carbo R. Antimicrobial and antioxidant activity of crude onion (Allium cepa, L.) extracts. Int J Food Sci Tech 2010;45:403-9.

24. Nitbani FO, Siswanta D, Solikhah EN. Isolation and antibacterial activity test of lauric acid from crude coconut oil (Cocos nucifera L.). Proc Chem 2016;18:132-40.

25. Pundir RK, Jain P. Evaluation of five chemical food preservatives for their antibacterial activity against bacterial isolates from bakery products and mango pickles. J Chem Pharm Res 2011;3:24-31.

26. Odiba JO, Musa AM, Hassan HS, Yahay SM, Okolo EI. Antimicrobial activity of isolated stigmast-5-en-3- $\beta$-ol $(\beta-$ sitosterol) from honeybee Propolis from North-Western, Nigeria. Int J Pharma Sci Res 2014;5:908-18.

27. Lekshmi PNCJ, Viveka S, Viswanathan MB, Manivannan G, Shobi TM. GC-MS Characterization of Volatile Odorous Compounds in Allium Cepa. Nanobiol Pharm Tech 2014;488-94. DOI:10. 13140/2.1.3278.7523.

28. Farag MA, Ali SE, Hodaya RH, El-Seedi HR, Sultani HN, Laub A, et al. Phytochemical profiles and antimicrobial activities of Allium cepa red cv. and Allium sativum subjected to different drying methods: a comparative MS-based metabolomics. Molecules 2017;22:1-18. 